\title{
Linked homozygous BMPR1B and PDHA2 variants in a consanguineous family with complex digit malformation and male infertility
}

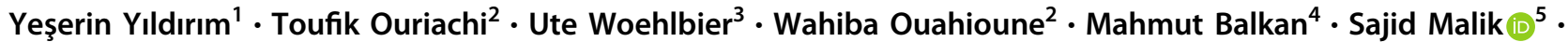 \\ Aslıhan Tolun $\mathbb{1}^{1}$
}

Received: 12 August 2017 / Revised: 30 November 2017 / Accepted: 1 February 2018 / Published online: 26 March 2018

(c) European Society of Human Genetics 2018

\begin{abstract}
In affected members of a consanguineous family, a syndrome, which is concurrence of set of medical signs, is often observed and commonly assumed to have arisen from pleiotropy, i.e., the phenomenon of a single gene variant affecting multiple traits. We detected six sibs afflicted with a unique combination of digit malformation that includes brachydactyly, symphalangism and zygodactyly plus infertility in males owing to azoospermia, sperm immotility or necrospermia, which we hypothesised to have arisen from a defect in a single gene. We mapped the disease locus and by exome sequencing identified in patients homozygous missense variants bone morphogenetic protein receptor type $I B(B M P R 1 B)$ c.640C $>\mathrm{T}$ (p. $(\operatorname{Arg} 214 C y s))$ and alpha-2 pyruvate dehydrogenase (PDHA2) c.679A $>\mathrm{G}$ (p.(Met227Val)). Structural protein modelling, protein sequence conservation and in silico analysis indicate that both variants affect protein function. BMPRIB is known to be responsible for autosomal dominant brachydactyly and autosomal recessive acromesomelic chondrodysplasia. Our findings show that also recessive complex digit malformation can be caused by BMPR1B variant and not all biallelic BMPRIB variants cause acromesomelic dysplasia. PDHA2 is a novel candidate gene for male infertility; the protein product is a mitochondrial enzyme with highest expression in ejaculated sperm. Our findings are a unique example of two linked variants, $\sim 711 \mathrm{~Kb}$ apart, in different genes that together manifest as a novel syndrome. They demonstrate that exome sequencing and not candidate gene approach should be employed in disease gene hunt, defining new diseases and genetic testing, to rule out the coincidental presence of two variants contributing together to the phenotype, which may be discerned as a novel disease.
\end{abstract}

These authors contributed equally: Yeşerin Yıldırım, Toufik Ouriachi.

Electronic supplementary material The online version of this article (https://doi.org/10.1038/s41431-018-0121-7) contains supplementary material, which is available to authorised users.

Sajid Malik

malik@qau.edu.pk

$\bowtie$ Aslihan Tolun

tolun@boun.edu.tr

1 Department of Molecular Biology and Genetics, Boğaziçi University, Istanbul, Turkey

2 Department of Pathology, University Hospital of Blida, Faculty of Medicine of Blida-1, Blida, Algeria

\section{Introduction}

Syndrome is a medical term meaning concurrence of set of medical signs. Aetiology of many syndromes has not been uncovered, and some syndromes might not even have genetic basis, e.g., foetal alcohol syndrome. Pleiotropy is the phenomenon of a single gene variant affecting multiple traits, and thus a gene variant with pleiotropic effect manifests as a syndrome. In a consanguineous family we

3 Center for Integrative Biology, Faculty of Science, Universidad Mayor, Santiago, Chile

4 Department of Medical Biology and Genetics, Faculty of Medicine, Dicle University, Diyarbakır, Turkey

5 Human Genetics Program, Department of Animal Sciences, Faculty of Biological Sciences, Quaid-i-Azam University, Islamabad, Pakistan 
observed digit malformation and male infertility, which we hypothesised as a new syndrome caused by pleiotropic effects of a single gene defect but instead found two linked variants in two different genes.

Acromesomelic chondrodysplasias (ACD) are characterised by shortening and malformation of the limbs, predominantly the forearms, forelegs, hands and feet, and generally manifest also with short stature. Inheritance is autosomal recessive. The majority of the cases result from GDF5 (growth/differentiation factor 5) variants, and four homozygous variants in BMPRIB (bone morphogenetic protein receptor type $I B$ ) also have been reported in ACD. The first such BMPRIB variant is severe and leads to acromesomelic dysplasia type Demirhan, similar to chondrodysplasia-type Grebe, caused by biallelic GDF5 variants [1]. The skeletal phenotype of the patient was aplasia of the fibula, severe brachydactyly, ulnar deviation of the hands, fusion of carpal/tarsal bones and disproportionate short stature, and the variant was homozygous 8-bp deletion c.361_368delGGACCTAT (p.(Gly121Thrfs*13)). The manifestations of two other variants, namely, c.157T $>C$ (p.(Cys53Arg)) and c.657G >A (p.(Trp219*)), are also like Grebe chondrodysplasia [2]. The last variant c.91C $>\mathrm{T}$ (p. (Arg31Cys)) was assessed to have a moderate impact on the protein and accordingly caused fibular hypoplasia and complex brachydactyly (du Pan dysplasia), a milder ACD [3]. The patient had disproportionate short stature, mild acromesomelic limb shortening, short fingers, abnormal finger joints, hypoplastic toes, misaligned ankle joints, and totally missing fibula. All these variants were identified by candidate gene approach. The novel homozygous BMPRIB variant in the consanguineous family we present does not cause ACD but instead a complex digit malformation including brachydactyly, symphalangism and zygodactyly. Not homozygous but heterozygous $B M P R I B$ variants are known to cause brachydactyly, either type A1 (BDA1D) or type A2 (BDA2).

Biallelic variants in several genes have been reported to underlie idiopathic azoospermia leading to male infertility (MIM 258150). Studies in mammals indicate that many more genes are involved in spermatogenesis (reviewed in ref [4]). One such gene is PDHA2 (alpha-2 pyruvate dehydrogenase), encoding the mitochondrial matrix enzyme PDHA2, which is expressed exclusively in testis and with maximal expression in ejaculated sperm [5, 6]. PDHA2 compensates for the absence of the X-linked PDHA1, which is not synthesised in sperm, thereby enabling vital energy metabolism [7]. PDHA1 and PDHA2 encode the E1-alpha subunits of pyruvate dehydrogenase complex in mitochondria, and two PDHA1 or PDHA2 peptides heterotetramerise with two PDHB peptides to form the enzyme. We identified a homozygous missense variant in PDHA2, which can account for the male infertility in the family.
Together, the BMPRIB and PDHA2 variants can explain the pathology in the family. The phenomenon of variants that affect protein function in more than one gene in the same individual is not very rare, but our finding of two such linked variants is unique.

\section{Materials and methods}

\section{Family}

The family is Algerian, and parents are first cousins. Seven (three males and four females) of the ten sibs were affected, but two sibs, one of them affected, had died in infancy due to fever and diarrhoea (Fig. 1a). Parents and all surviving sibs participated in the study. They were physically examined, and their photographs were taken. Roentgenograms of three affected and two unaffected sibs were acquired.

Written informed consent was obtained from all participants, and the study protocol was approved by the Ethical Review Committee of Quaid-i-Azam University and the Boğaziçi University Institutional Review Board for Research with Human Participants.

\section{Genetic analysis}

DNA samples of the mother and four affected and two unaffected sibs were available for the genetic study (Fig. 1a). They were subjected to SNP genotyping with Illumina Chip Human OmniExpress-24 containing $>720 \mathrm{~K}$ markers. The Illumina Genome Studio (v1.02) Genotyping Module was used to handle the genotype data. Linkage analysis was performed with Allegro software v1.2c in EasyLinkage v5.08 platform. Markers were selected at an interval of $0.01 \mathrm{Mb}$ and used in blocks of 30. An autosomal recessive inheritance model with full penetrance and a disease allele frequency of 0.001 were assumed. SNP data were arranged in MS-Excel to compare genotypes and to investigate for shared homozygosity in the affected sibs at the candidate loci. Duplication-deletion analysis was performed in Genome Studio v3.2.0 using the cnvPartition copy number variation Analysis Plug-in. Another linkage analysis was performed similarly, with the genotype data of the mother and sons only to search for the male infertility locus.

DNA sample of affected sib 412 was subjected to exome sequencing with the SeqCap EZ MedExome kit on an Illumina HiSeq2500 (paired-end $2 \times 75 \mathrm{bp}$ ) with an average coverage of $10 \times$ for $95 \%$ of the bases targeted. Seventy-six percent of the covered regions had at least $20 \times$ coverage. A minimum of $10 \times$ coverage was achieved for $94 \%$ and $93 \%$ for the larger and the smaller candidate loci, respectively. Raw reads were aligned to reference human genome hg19 
A

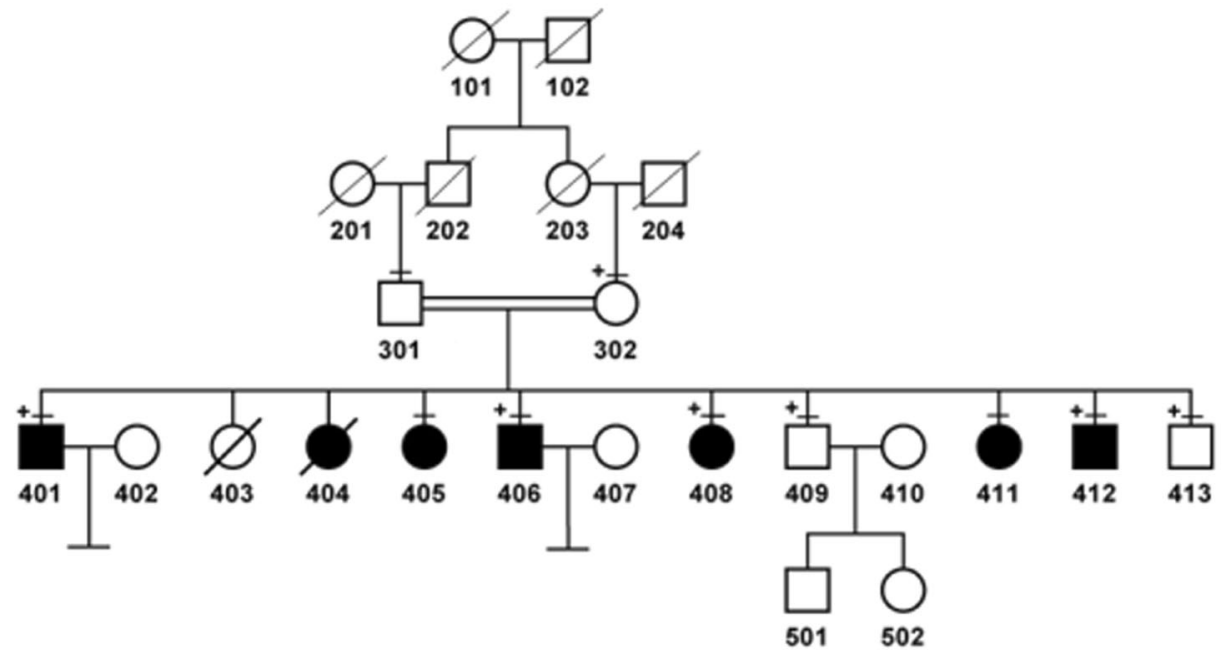

B

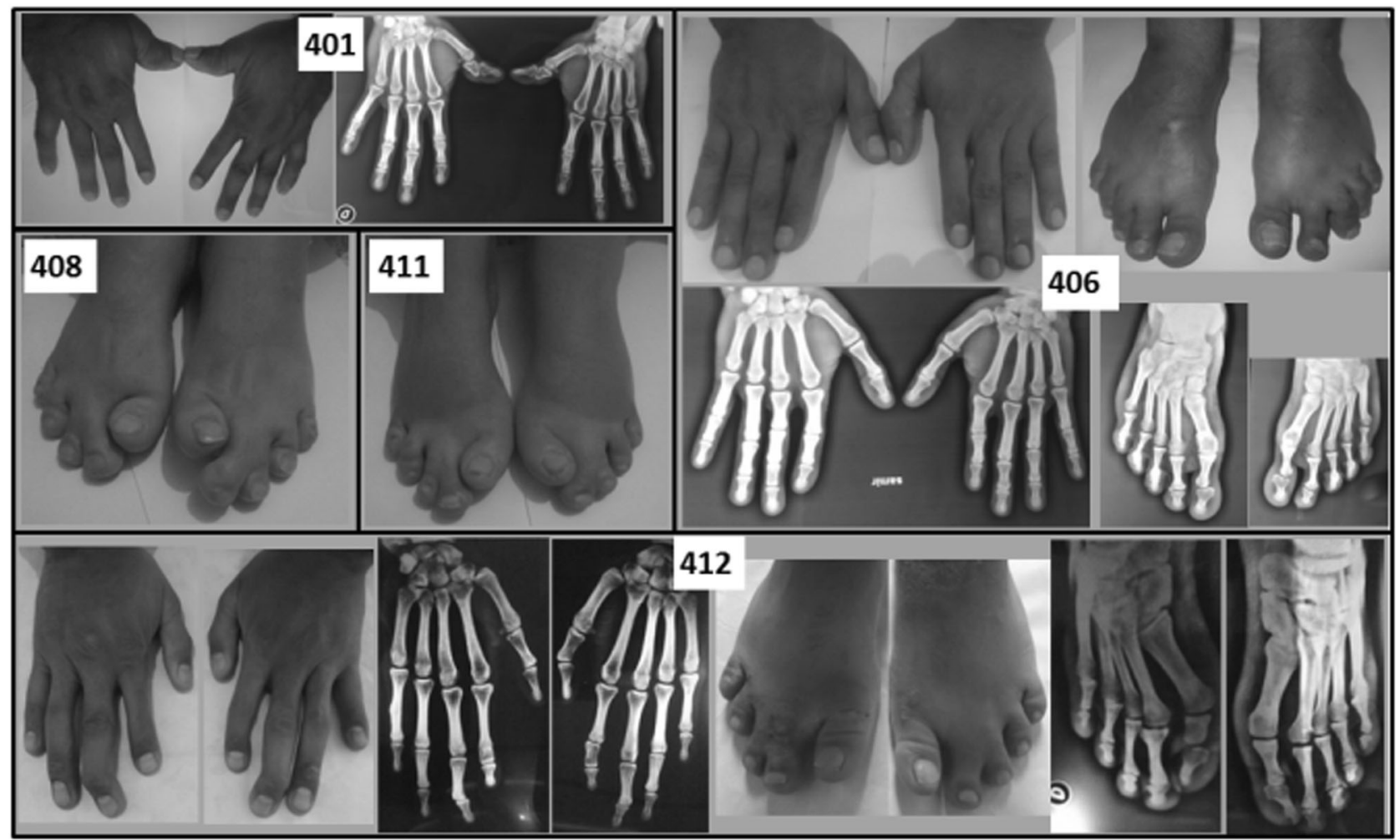

Fig. 1 Family. a Pedigree. Individuals included in the genetic study are indicated by + . A horizontal line above a symbol indicates that the individual has been clinically evaluated. b Photographs and roentgenograms of the affected siblings

(GRCh37) using BWA-0.5.9. Removal of duplicates as well as variant calling was performed with SAMtools-0.1.14, and ANNOVAR [8] was used to annotate variants. Variants residing within the candidate chromosomal regions were considered, and those with read depths $<0.60$ of the total read depth and minor allele frequencies $>0.01$ as reported in any population in the Exome Aggregation Consortium (ExAC) or 1000 Genomes databases were filtered out. Aligned reads of the exome data were analysed with
Integrative Genomics Viewer (IGV) v2.3 to investigate whether all coding exons of the candidate genes in the candidate regions had been covered in the exome sequencing. Additionally, all candidate variants were investigated for verification with the same software. To double check, all possibly rare homozygous variants affecting function throughout the whole genome were filtered, and whether they were in a region of shared homozygosity was investigated. The variants in $B M P R 1 B, P D H A 2$ and $A N K 2$ are 
submitted to ClinVar (www.ncbi. nlm.nih.gov/clinvar/) with submission numbers SUB2781172, SUB2769712 and SUB2837519, respectively. Also, for the seven individuals whose DNA samples were available the phenotypes and genotypes with respect to those variants are submitted to database LOVD (www.LOVD.nl/CAV3) with individual IDs 133223, 133226-133230 and 107535.

Online prediction tools MutationTaster2, SIFT, PolyPhen-2, LRT, FATHMM, RadialSVM and LR were employed to predict damage of the variants to the protein [9-14]. The two strong candidate variants were validated by Sanger sequencing, and that they segregated with the disease was ascertained by single-strand conformational polymorphism analysis (SSCP). In addition, 42 azoospermic and 15 oligospermic men were screened for variants in PDHA2 by SSCP. The subjects have been described previously [15].

HomoloGene was used to evaluate the conservation status of the residues altered by the candidate variants [16], and the alignment figures were prepared using Geneious v10.0.5 [17]. Gene expression information for the candidate disease genes was obtained from Expression Atlas (www. ebi.ac.uk/gxa/home) and published reports.

\section{Structural analysis}

Sequence alignment was performed using online tool PRALINE [18]. Structural analysis was performed using the SWISSMODEL server (http://swissmodel.expasy.org/) and Chimera1.11rc imaging software. The Protein Data Bank (PDB) structures 3mdy.1.A of wild-type BMPR1B and 3exe.1.A of wild-type PDHA2 were used as templates to model the structures of BMPR1B p.Arg214Cys and PDHA2 p.Met227Val variants, respectively (http://www. rcsb.org/pdb/explore.do?structureId = 3MDY).

\section{Results}

\section{Clinical findings}

Affected sibs had a unique combination of digit malformations characterised by brachydactyly and camptodactyly of certain digits in hands and feet, symphalangism of some fingers, and zygodactyly in feet. The findings are compiled in Table 1. The conspicuous features present in four or more affected subjects were symphalangism of index fingers at proximal-phalangeal joint (PPJ), short and broad hallux, cutaneous fusion of 2-3 toes and short fifth toe. In addition, affected sibs had other malformations in different combinations: short and/or symphalangus thumb, camptodactylous and/or symphalangus third finger, short and/or symphalangus fifth finger, crowding of carpals and high digits-to-palm length ratio, and in feet valgus and/or short and broad hallux (Fig. 1b). Various other features were observed in single sibs only, including camptodactylous fifth finger and symphalangism at distal phalangeal joint of fourth finger in 401, camptodactylous 3-4 toes in 406, symphalangus fifth finger in 408, and short and symphalangus fifth finger in 412. The two brothers we considered not affected had only minor findings in hands and feet.

Radiographic evaluation of zeugopod and stylopod limb segments of patient 412 revealed that the lengths of the long bones were within normal ranges and did not present evidence for fusion of radius/ulna or tibia/fibula or bowing of long bones (Supplementary Figure 1), excluding the possibility of a very mild form of acromesomelic chondrodysplasia.

The three affected brothers additionally had sperm anomalies, either azoospermia or oligospermia with dead sperm (necrospermia) and sperm with decreased motility or total immotility (Table 2). Two of them were married for 8 and 5 years without children. Sperm analysis was performed also for unaffected brother 409 , and the result was mild necrospermia. Blood hormone levels investigated for affected sister 408 were normal (Supplementary Table 1).

All affected sibs had normal height (Table 1) and normal mental status. None had any craniofacial signs except for down-slanting eyes, a feature also observed in the two unaffected sibs. Two affected and four unaffected members of the family had recurrent styes. These two findings are likely not related to the disease in the family.

\section{Genetic findings}

We performed linkage analysis using the SNP data of six family members (Supplementary Figure 2). Maximal multipoint logarithm of the odds (LOD) scores of 4.25 and 3.26 were obtained at two loci $>1 \mathrm{Mb}$ where homozygous genotypes were shared by all affected subjects and no other member of the family. At both loci the haplotypes had likely descended from a recent common ancestor. The loci were $<4-\mathrm{Mb}$ apart, interrupted by two ancestral crossovers. We considered both loci as candidates to harbour the variant underlying the disease afflicting the family: the $\sim 18.5-\mathrm{Mb}$ maximal region at 4q23-27 between rs230489 (nucleotide $103,388,441)$ and $\operatorname{rs} 11098592(121,888,708)$ and the 4.3$\mathrm{Mb}$ region at 4q22.2-22.3 between rs4699852 $(95,342,789)$ and rs7680513 $(99,608,363)$.

At the candidate loci, the strongest candidate variants were $B M P R 1 B$ c.640C $>\mathrm{T}$ (p.(Arg214Cys); NM_001203.2) and PDHA2 c.679A $>\mathrm{G}$ (p.(Met227Val); rs200969445; NM_005390.1), both at the larger locus (Supplementary Table 2). The former variant is novel, and the latter is reported in ExAC in Latino and non-Finnish European populations only, with frequencies of 0.00009 ( 1 in 11570 alleles) and 0.00006 (4 in 66718 alleles), respectively. All 


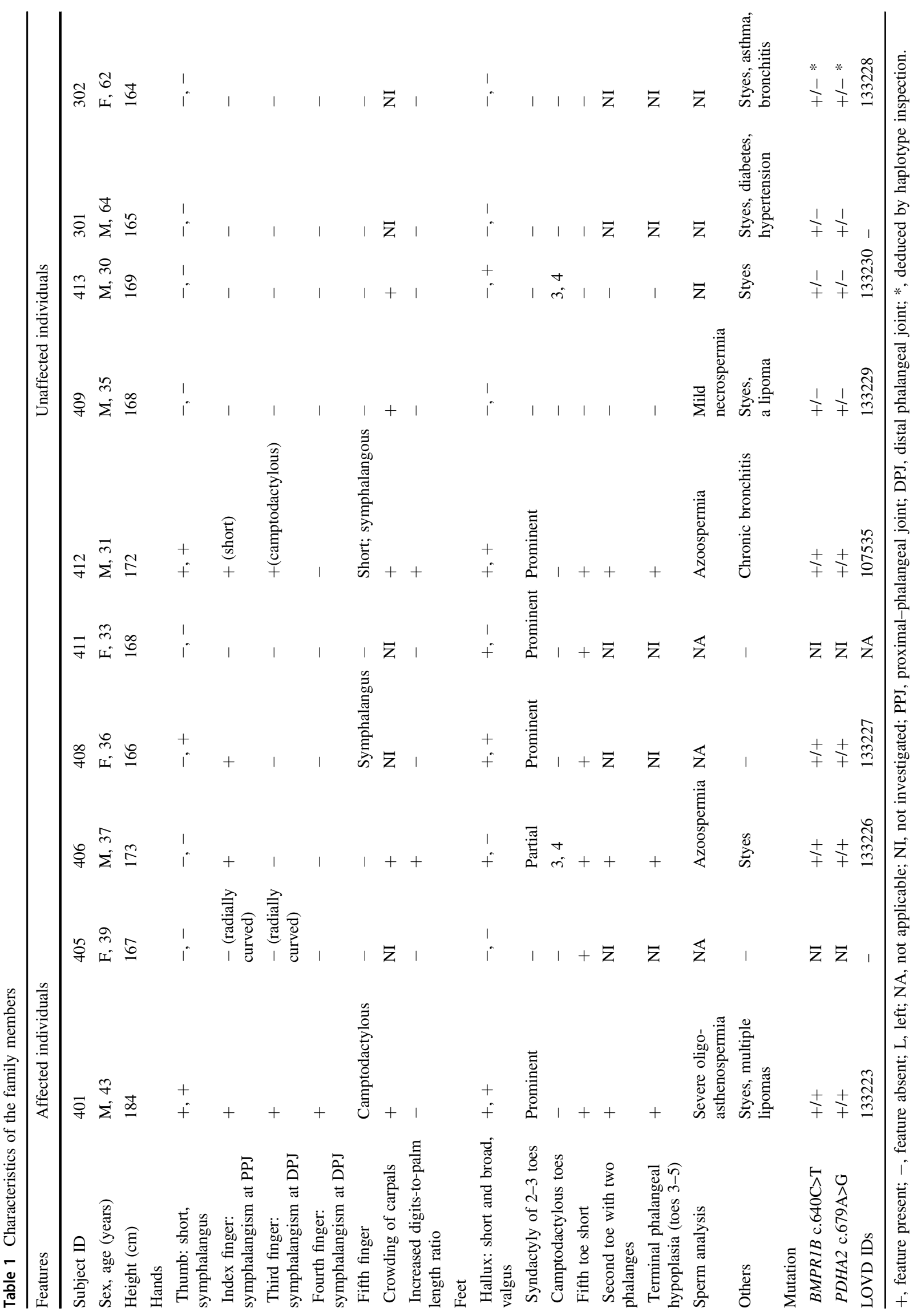


Table 2 Semen analysis and spermiograms of the brothers

\begin{tabular}{|c|c|c|c|c|c|}
\hline Findings & 401 & 406 & 412 & 409 & Reference \\
\hline PDHA2 c. $679 \mathrm{~A}>\mathrm{G}$ & $+/+$ & $+/+$ & $+/+$ & $+1-$ & $-1-$ \\
\hline Volume (ml) & 3.0 & 1.4 & 2.6 & 2.5 & $>1.5$ \\
\hline Appearance & Opaqueness decreased, grey & $\begin{array}{l}\text { Opaqueness } \\
\text { decreased, white }\end{array}$ & $\begin{array}{l}\text { Opaqueness } \\
\text { decreased, white }\end{array}$ & Opaque, grey & Opaque, grey \\
\hline $\mathrm{pH}$ & 8.0 & 8.5 & 8.5 & 8.0 & $>7.2$ \\
\hline Viscosity & Normal & Decreased & Decreased & Normal & Semi-liquid \\
\hline Spermatozoa (million/ml) & 1.6 & 0 & 0 & 74.8 & $>15$ \\
\hline Total spermatozoa (million) & 4.8 & 0 & 0 & 187 & $>39$ \\
\hline Round cells & 0.0019 & - & - & 1.02 & $<10$ \\
\hline \multicolumn{6}{|l|}{ Motility after $1 \mathrm{~h}^{\mathrm{a}}(\%)$} \\
\hline Progressive $(a+b)$ & 12 & - & - & 53 & 32 \\
\hline Non-progressive (c) & 4 & - & - & 4 & 8 \\
\hline Total motility $(a+b+c)$ & 16 & - & - & 57 & $>40$ \\
\hline Motionless (d) & 84 & - & - & 43 & $<40$ \\
\hline \multicolumn{6}{|l|}{ Motility after $3 \mathrm{~h}^{\mathrm{a}}(\%)$} \\
\hline Progressive $(a+b)$ & 8 & - & - & 40 & $\leq 50 \%(\mathrm{e})$ \\
\hline Non-progressive (c) & 3 & - & - & 3 & $\leq 50 \%(\mathrm{e})$ \\
\hline Total motility $(a+b+c)$ & 12 & - & - & 43 & $\leq 50 \%(\mathrm{e})$ \\
\hline Motionless (d) & 88 & - & - & 57 & $40-50$ \\
\hline \multicolumn{6}{|l|}{ Spermiogram } \\
\hline Vitality (\%) & 28 & - & - & 52 & $>58$ \\
\hline Agglutinates & Absent & - & - & Absent & Absent \\
\hline Typical sperm (\%) & 29 & - & - & 50 & $>4$ \\
\hline Sperm with defects $(\%)$ & 71 & - & - & 40 & $<96$ \\
\hline Head & 48 & - & - & 26 & $<35$ \\
\hline intermediate piece & 0 & - & - & 16 & $<20$ \\
\hline Flagellum & 33 & - & - & 10 & $<20$ \\
\hline Cytoplasm & 0 & - & - & 13 & $<20$ \\
\hline Total defective sperms & 81 & - & - & 65 & $<85$ \\
\hline Teratospermia index & 1.14 & - & - & 1.62 & $<1.46$ \\
\hline Polynuclear (million/ml) & 0.00033 & - & - & 1.2 & $<0.001$ \\
\hline Other cells & - & - & - & $\begin{array}{l}\text { Few epithelial } \\
\text { cells }\end{array}$ & $\begin{array}{l}\text { White blood cells } \\
(<1 \text { million/ml })\end{array}$ \\
\hline Diagnosis & $\begin{array}{l}\text { Severe oligo-asthenospermia; } \\
\text { necrospermia }\end{array}$ & $\begin{array}{l}\text { Total } \\
\text { azoospermia }\end{array}$ & Total azoospermia & Mild necrospermia & - \\
\hline
\end{tabular}

a, rapid and progressive motility; b, slow and gradual motility; c, motility on site; d, immotile; e, decreased as compared with first hour.

Reference: World Health Organisation: Laboratory Manual for the Examination and Processing of Human Semen 2010.

seven online tools predicted both variants to be diseasecausing (Supplementary Table 2). The variants were validated by Sanger sequencing (Supplementary Figure 3) and ascertained to segregate with the disease in the family. All affected sibs were homozygous for the variants, whereas mother, father (as deduced) and unaffected brothers were heterozygous for both.

To ascertain that there was no other candidate locus for male infertility, we performed a linkage analysis using the genotype data of the mother and sons only. We detected again the same locus, with a maximal LOD score of 2.65 .
All other loci where the infertile brothers shared homozygosity were $<0.75 \mathrm{Mb}$.

Not to miss any variants possibly contributing to the disease aetiology, we considered additionally all regions with high LOD scores and sizes $>200 \mathrm{~kb}$ obtained in the two linkage analyses and did not find a good candidate variant in any.

No rare (frequency $<0.14$ ) exonic or splicing variant was detected in the known brachydactyly-syndactyly genes HOXD13, NOG, IHH, BMP2, GDF5, PTHLH and ROR2 (data not shown). 


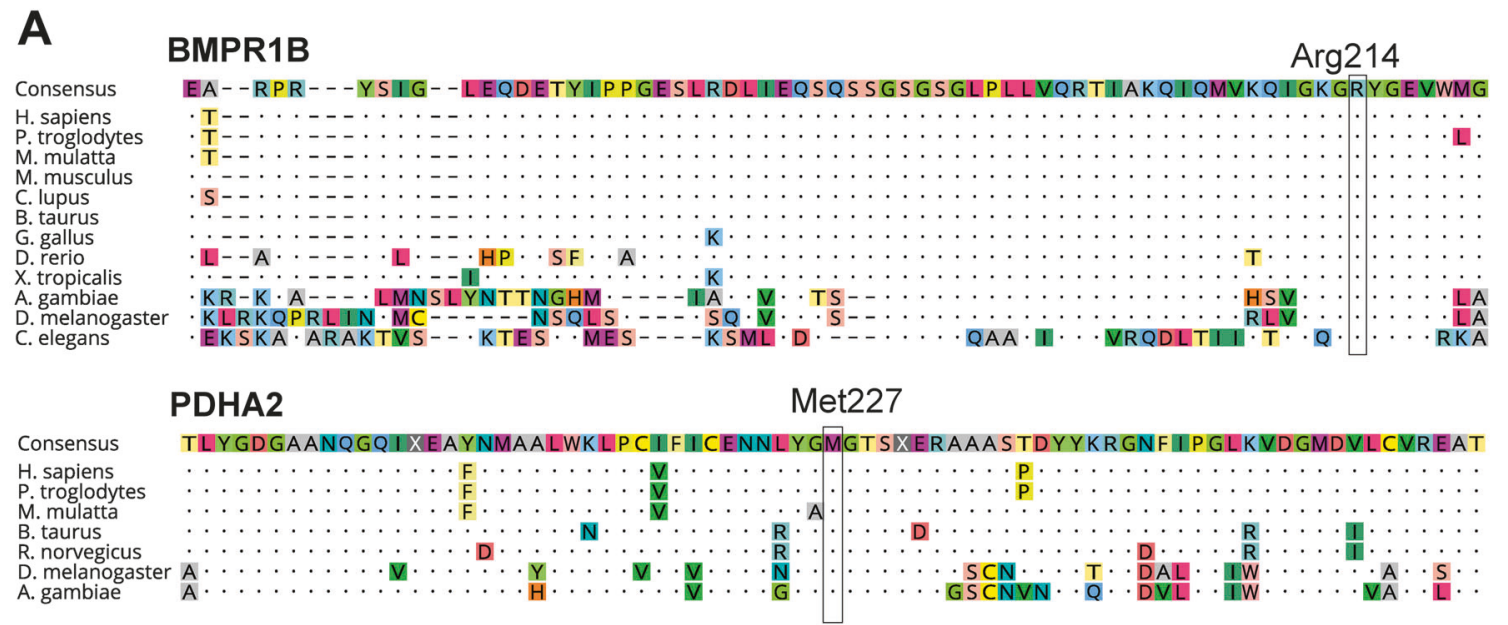

B
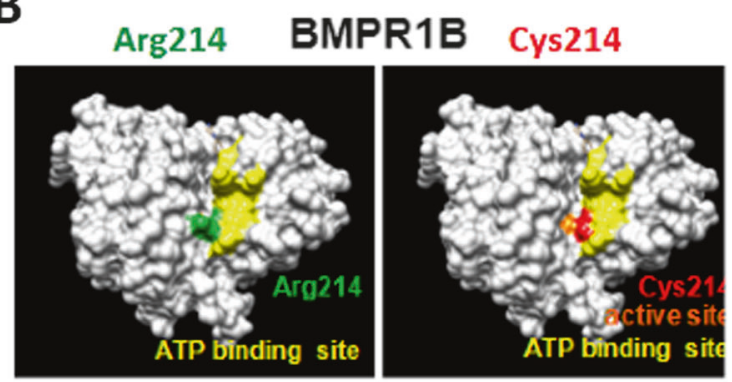

D
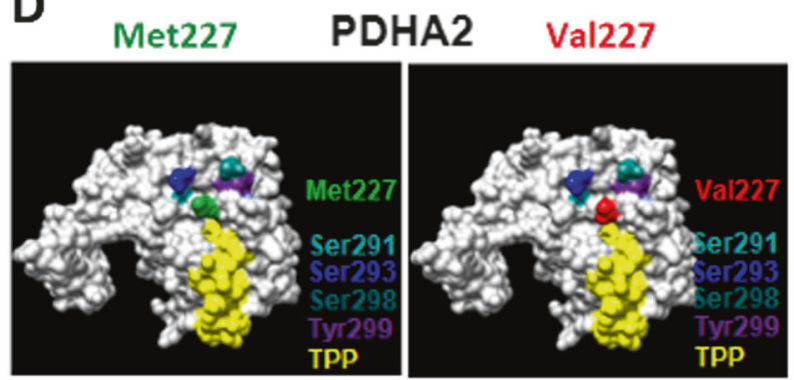

Fig. 2 Amino-acid sequence conservation and predicted effects of the variants on the proteins. a Sequence conservation for altered residues (boxed) in BMPR1B and PDHA2. b Surfaces of wild-type and variant BMPR1B. The location of wild-type Arg214 is indicated in green and of substituted Cys214 in red. c An overlay of the backbone structures of wild-type and variant BPMR1B. The change to a Cys at residue 214 causes a minor shift in the backbone position as indicated by a red arrow and red marking of the backbone, whereas the backbone position in the wild-type is indicated by a green arrow and green marking.

We did not detect any rare PDHA2 variant in the 57 azoo/oligospermic men we screened. The lowest frequency for any homozygous variant was $>0.164$.

\section{Protein modelling}

We found that both variants alter residues that are highly conserved across species (Fig. 2a) and performed structural modelling for the proteins. BMPR1B Arg214 is located
C

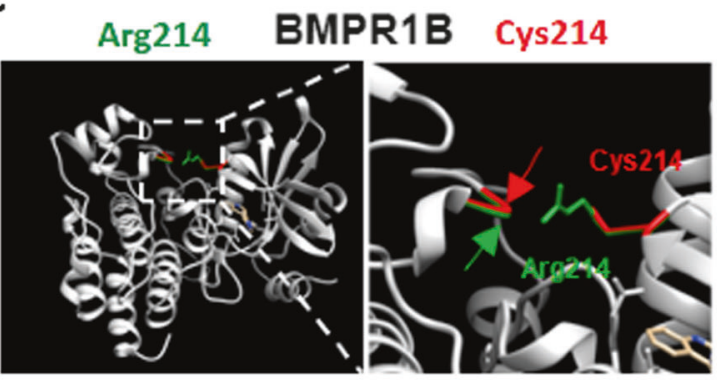

E

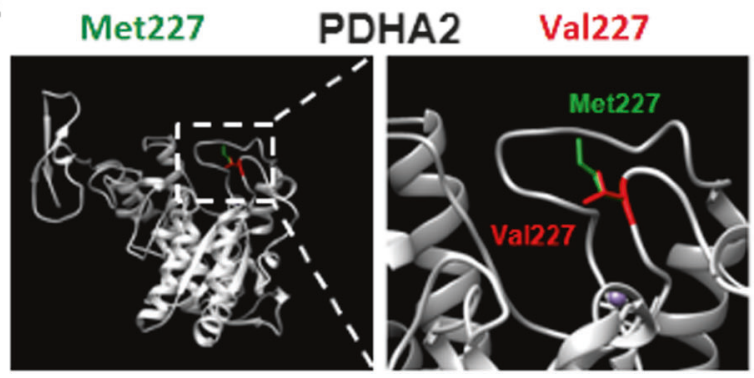

Substituted residue 214 locates within the ATP-binding site (yellow) and proximal to the active site (orange). $\mathbf{d}$ Surfaces of wild-type and variant PDHA2. Residue 227 is surrounded by known (Ser291 and Ser298) [20] and predicted (Ser293 and Tyr299) phosphorylation sites, highlighted in various shades of blue as well as a TPP-binding motif, highlighted in yellow. e An overlay of the backbone structures of wildtype and variant PDHA2. The location of wild-type Met227 is indicated in green and substituted Val227 in red.

within the cytoplasmic protein kinase domain of the transmembrane protein and in a putative ATP binding motif spanning residues 210-218. In the three dimensional structure, it is in close proximity to the active site residue 332 (Fig. 2b). Arginine has a long side chain, which seems to cover the active site, whereas the substituted cysteine is smaller and exposes the active site (Fig. 2c). This change in active site accessibility may alter BMPR1B activity [19]. Considering together all known variants, we did not find 
any correlation with the topologic location of the variants and the disease type (Supplementary Figure 4A).

Altered residue Met227 in PDHA2 is surrounded by known phosphorylation sites targeted by the PDK family of kinases (Fig. 2d,e) [20]. Phosphorylation at Ser291 interferes with access to the active site, resulting in the inactivation of the enzyme, and dephosphorylation of all three serines (Ser230, Ser291 and Ser298) is required for reactivation. We speculate that the substitution of Met227 with valine could affect the phosphorylation status of PDHA2 and thereby modulate the activity of the protein. Moreover, the position of the altered residue is proximal to a thiamine pyrophosphate (TPP)-binding motif (Fig. 2d), and hence the alteration could affect the binding of TPP, a cofactor of pyruvate dehydrogenases [21].

\section{Discussion}

The presented family exhibits a unique combination of digit anomalies and male infertility, distinct from known syndromes. The herein identified linked variants that are only $711 \mathrm{~Kb}$ apart can together explain the phenotype in the family, indicating coincidental presence of two unique diseases in the affected family members. The BMPRIB variant could account for the complex digit malformation and the PDHA2 variant for the male infertility. Both the digit malformation and male infertility phenotype were variable in the homozygotes (Tables 1 and 2). In some but not all heterozygotes much milder forms of the traits were seen. BMPRIB is already associated with bone dysmorphologies [1-3], but PDHA2 has not been associated with any disease. The loci for syndromes manifesting with digit anomalies and infertility together, such as DauwersePeters syndrome (MIM 611733; $\mathrm{t}(4 ; 6)(\mathrm{q} 12 ; \mathrm{p} 23)$ ), deafnessinfertility syndrome (MIM 611102; 15q15.30), and short stature, onychodysplasia, facial dysmorphism and hypotrichosis syndrome (SOFT; MIM 614813; 3p21) are all excluded by linkage analysis.

The six affected sibs had similar digit malformations but with some variations. The two heterozygous brothers (409 and 413) also had digit anomalies, albeit very mild (Table 1). All three affected males had infertility problems, again variable; the condition was more severe in two of them, with no sperm in the ejaculate, and in the other the few sperms in the ejaculate were either dead or immotile. It was not surprising that some of the sperms of the heterozygous brother investigated (409) were dead, as half of his spermatids would have defective PDHA2. Normal hormone levels in affected sister 408 excluded ovarian dysfunction.

Both causative variants found in the family are missense and predicted to affect protein function, and amino-acid sequences are conserved among species with bilateral symmetry. Affected residue Arg214 in BMPR1B is conserved among all 98 vertebrate sequences available in the UCSC Genome Browser (data not shown), and 64 residues around the residue are completely conserved among six mammals (HomoloGene) (Fig. 2a). Arginine is hydrophilic and the most strongly positively charged of all amino acids, whereas sulphur-containing cysteine is not hydrophilic; moreover, disulphide-bonding cysteine has strong hydrophobic properties and is neutral. The pairwise identity for the entire amino-acid sequence of the protein between human and chimpanzee is $99.1 \%$, whereas it is 98.7 and 92.7\% among mammals and vertebrates, respectively. Altered PDHA2 residue Met227 is conserved among 94 of the 95 vertebrates available in UCSC Genome Browser (data not shown). The surrounding tyrosine residues are also conserved among mammals and known to be of importance for its enzymatic function [22, 23]. Both methionine and valine are uncharged hydrophobic amino acids, but valine is so to a much greater extent; moreover, methionine is a sulphur-containing amino acid. The pairwise identity for the entire amino acid sequence of the protein is $99.7 \%$ between human and chimpanzee, whereas it is 85.5 and $84.2 \%$ among mammals and vertebrates, respectively. Evaluation of the three dimensional structures of the variant proteins indicated that the substitutions could affect the enzymatic activity of the respective proteins and thereby impair their functions [20, 22-24].

The patients in the presented family were of normal height, and the radiological evaluation of long bones was not indicative of mesomelic or acromelic dwarfism. In contrast, all four homozygous $B M P R 1 B$ variants reported to date are known to cause ACD [1-3]. Additional features were aplasia of the fibula [1], absence of the fibula [2, 3], bilateral clubfoot deformity [1] and short stature [1, 2]. Also, brachydactyly in the patients we present is much milder, restricted to only a few phalanges as opposed to all extremely short digits in the reported cases, and is accompanied by other clinical features such as coetaneous syndactyly in feet, symphalangism in hands, and terminal phalangeal hypoplasia of postaxial toes. The two heterozygous sibs but not the parents exhibited very mild digit malformations. None of the heterozygotes for BMPRIB variants with recessive effect were reported to have any phenotypic features $[1,2]$.

As for BMPRIB variants with dominant effect, in total six have been reported (Supplementary Figure 4B) [1-3, 25-27]. They cause milder disorders, either brachydactyly type A1 or type A2. The homozygous BMPR1B missense variant in the family we present contrasts with those variants in the sense that (1) although it resides in the catalytic domain, as does another missense variant Lys325Asn, it is disease-causing mainly in the homozygous state, (2) it manifests with brachydactyly as do the variants with 
dominant effect but with additional digit anomalies, and (3) in homozygous state, it does not lead to ACD. Also, in contrast, all other 12 isolated brachydactyly entries listed in OMIM are caused by heterozygous variants, albeit in other genes.

The male infertility trait in the family is compatible with PDHA2 deficiency. The few sperm in the ejaculate seemed to have developed fully but not have had the energy to survive the journey to ejaculation. In such a situation it would be expected that the majority of the sperms would degenerate in the epididymides, and those that make it to ejaculation are either dead or immotile. The condition is like 'epididymal necrospermia' described in four men with oligospermia and necrospermia; sperm death owing to degeneration was proposed to occur in the epididymis, as testicular sperm were ultrastructurally normal [28]. In three of those men, frequent ejaculation was practiced to deplete epididymal sperm reserves and reduce the period spent in the epididymis, resulting in three- to sevenfold increase in sperm motility. PDHA2 is a mitochondrial enzyme, and azoospermia, asthenozoospermia (reduced sperm motility) and necrospermia are all compatible with deficiency of PDHA2. To date necrospermia and asthenozoospermia have been associated mainly with mtDNA polymorphisms and deletions [29-32]. Considering also that the PDHA2 variant we identified resides at the mapped locus for the trait and it was assessed as affecting function, we propose that the variant most probably underlies the infertility in the brothers. Thus, we screened other 57 azoo/oligospermic men for variants in the gene but did not detect any rare biallelic variants. We had not found any possibly causative $T A F 4 B$ or ZMYND15 variant in those men, either [15]. Generally, only a few unrelated azoospermic men are reported to have variants in each of the known recessive genes for azoospermia [15, 33, 34].

In summary, our results show that (i) a homozygous $B M P R 1 B$ variant underlies a complex and recessive digit malformation, (ii) PDHA2 is a novel candidate gene for male infertility and (iii) concurrence of a unique set of medical signs (syndrome) can be caused by two linked variants in different genes even in all affected members of a family. To our knowledge, the presented family is the first with two such co-segregating variants leading to the coincidental occurrence of two different diseases. Our findings raise caution in identifying new disease genes and defining new syndromes or new phenotypes for disease genes, and emphasise that exclusion of a second contributing gene defect is essential.

Acknowledgements We thank the family members for their cooperation. This study was supported by Scientific and Technologic Research Council of Turkey (TÜBİTAK) grant $114 Z 829$ and Boğaziçi University Research Fund grant 10860. UW is supported by FONDECYT no. 1150743.
Compliance with ethical standards The study protocol was approved by the Ethical Review Committee of Quaid-i-Azam University and the Boğaziçi University Institutional Review Board for Research with Human Participants.

Conflict of interest The authors declare that they have no conflict of interest.

\section{References}

1. Demirhan O, Turkmen S, Schwabe GC, et al. A homozygous BMPR1B mutation causes a new subtype of acromesomelic chondrodysplasia with genital anomalies. J Med Genet. 2005;42:314-7.

2. Graul-Neumann LM, Deichsel A, Wille U, et al. Homozygous missense and nonsense mutations in BMPR1B cause acromesomelic chondrodysplasia-type Grebe. Eur J Hum Genet. 2014;22:726-33.

3. Stange K, Désir J, Kakar N, et al. A hypomorphic BMPR1B mutation causes du Pan acromesomelic dysplasia. Orphanet J Rare Dis. 2015;10:1.

4. Chalmel F, Rolland AD. Linking transcriptomics and proteomics in spermatogenesis. Reproduction. 2015;150:R149-R157.

5. Dahl H-H, Brown R, Hutchison W, Maragos C, Brown G. A testis-specific form of the human pyruvate dehydrogenase E1 $\alpha$ subunit is coded for by an intronless gene on chromosome 4 . Genomics. 1990;8:225-32.

6. Pinheiro A, Silva MJ, Graca I, et al. Pyruvate dehydrogenase complex: mRNA and protein expression patterns of E1 alpha subunit genes in human spermatogenesis. Gene. 2012;506:173-8.

7. Ficarro S, Chertihin O, Westbrook VA, et al. Phosphoproteome analysis of capacitated human sperm evidence of tyrosine phosphorylation of a kinase-anchoring protein 3 and valosincontaining protein/p97 during capacitation. J Biol Chem. 2003;278:11579-89.

8. Chang X, Wang K. wANNOVAR: annotating genetic variants for personal genomes via the web. J Med Genet. 2012;49:433-6.

9. Chun S, Fay JC. Identification of deleterious mutations within three human genomes. Genome Res. 2009;19:1553-61.

10. Dong C, Wei P, Jian X, et al. Comparison and integration of deleteriousness prediction methods for nonsynonymous SNVs in whole exome sequencing studies. Hum Mol Genet. 2015;24:2125-37.

11. Shihab HA, Gough J, Cooper DN, Day IN, Gaunt TR. Predicting the functional consequences of cancer-associated amino acid substitutions. Bioinformatics. 2013;29:1504-10. btt182

12. Sim N-L, Kumar P, Hu J, Henikoff S, Schneider G, Ng PC. SIFT web server: predicting effects of amino acid substitutions on proteins. Nucleic Acids Res. 2012;40:W452-W457.

13. Schwarz JM, Cooper DN, Schuelke M, Seelow D. MutationTaster2: mutation prediction for the deep-sequencing age. Nat Methods. 2014;11:361-2.

14. Adzhubei IA, Schmidt S, Peshkin L, et al. A method and server for predicting damaging missense mutations. Nat Methods. 2010;7:248-9.

15. Ayhan Ö, Balkan M, Guven A, et al. Truncating mutations in TAF4B and ZMYND15 causing recessive azoospermia. J Med Genet. 2014;51:239-44.

16. Zhang Z, Schwartz S, Wagner L, Miller W. A greedy algorithm for aligning DNA sequences. J Comput Biol. 2000;7:203-14.

17. Kearse M, Moir R, Wilson A, et al. Geneious Basic: an integrated and extendable desktop software platform for the organization and analysis of sequence data. Bioinformatics. 2012;28:1647-9.

18. Simossis VA, Heringa J. PRALINE: a multiple sequence alignment toolbox that integrates homology-extended and secondary structure information. Nucleic Acids Res. 2005;33:W289-W294. 
19. Kato M, Wynn RM, Chuang JL, et al. Structural basis for inactivation of the human pyruvate dehydrogenase complex by phosphorylation: role of disordered phosphorylation loops. Structure. 2008;16:1849-59.

20. Korotchkina LG, Sidhu S, Patel MS. Characterization of testisspecific isoenzyme of human pyruvate dehydrogenase. J Biol Chem. 2006;281:9688-96.

21. Muller YA, Lindqvist Y, Furey W, Schulz GE, Jordan F, Schneider G. A thiamin diphosphate binding fold revealed by comparison of the crystal structures of transketolase, pyruvate oxidase and pyruvate decarboxylase. Structure. 1993;1:95-103.

22. Blom N, Sicheritz-Pontén T, Gupta R, Gammeltoft S, Brunak S. Prediction of post-translational glycosylation and phosphorylation of proteins from the amino acid sequence. Proteomics. 2004;4:1633-49.

23. Kumar V, Rangaraj N, Shivaji S. Activity of pyruvate dehydrogenase A (PDHA) in hamster spermatozoa correlates positively with hyperactivation and is associated with sperm capacitation. Biol Reprod. 2006;75:767-77.

24. Kolobova E, Tuganova A, Boulatnikov I, Popov KM. Regulation of pyruvate dehydrogenase activity through phosphorylation at multiple sites. Biochem J. 2001;358:69-77.

25. Badura-Stronka M, Mroz D, Beighton $\mathrm{P}$, et al. Novel mutation in the BMPR1B gene (R486L) in a polish family and further delineation of the phenotypic features of BMPR1B-related brachydactyly. Birth Defects Res Part A-Clin Mol Teratol. 2015;103:567-72.
26. Lehmann K, Seemann P, Stricker S, et al. Mutations in bone morphogenetic protein receptor $1 \mathrm{~B}$ cause brachydactyly type A2. Proc Natl Acad Sci USA. 2003;100:12277-82.

27. Racacho L, Byrnes AM, MacDonald H, et al. Two novel diseasecausing variants in BMPR1B are associated with brachydactyly type A1. Eur J Hum Genet. 2015;23:1640-5.

28. Wilton LJ, Temple-Smith PD, Baker HW, de Kretser DM. Human male infertility caused by degeneration and death of sperm in the epididymis. Fertil Steril. 1988;49:1052-8.

29. Holyoake AJ, McHugh P, Wu M, et al. High incidence of single nucleotide substitutions in the mitochondrial genome is associated with poor semen parameters in men. Int $\mathbf{J}$ Androl. $2001 ; 24: 175-82$

30. Kao SH, Chao HT, Liu HW, Liao TL, Wei YH. Sperm mitochondrial DNA depletion in men with asthenospermia. Fertil Steril. 2004;82:66-73.

31. St John JC, Jokhi RP, Barratt CLR. The impact of mitochondrial genetics on male infertility. Int J Androl. 2005;28:65-73.

32. Thangaraj K, Joshi MB, Reddy AG, Rasalkar AA, Singh L. Sperm mitochondrial mutations as a cause of low sperm motility. J Androl. 2003;24:388-92.

33. Avenarius MR, Hildebrand MS, Zhang Y, et al. Human male infertility caused by mutations in the CATSPER1 channel protein. Am J Hum Genet. 2009;84:505-10.

34. Maor-Sagie E, Cinnamon Y, Yaacov B, et al. Deleterious mutation in SYCE1 is associated with non-obstructive azoospermia. $\mathrm{J}$ Assist Reprod Genet. 2015;32:887-91. 